\title{
Superconducting Pairing Symmetries in Anisotropic Triangular Quantum Antiferromagnets
}

\author{
J. Y. Gan ${ }^{1}$, Yan $\mathrm{Chen}^{2}$, and F. C. Zhang ${ }^{21}$ \\ ${ }^{1}$ Center for Advanced Study, Tsinghua University, Beijing, 100084, China \\ ${ }^{2}$ Department of Physics and Center of Theoretical and Computational Physics, \\ The University of Hong Kong, Hong Kong, China
}

\begin{abstract}
Motivated by the recent discovery of a low temperature spin liquid phase in layered organic compound $\kappa$-(ET) ${ }_{2} \mathrm{Cu}_{2}(\mathrm{CN})_{3}$ which becomes a superconductor under pressure, we examine the phase transition of Mott insulating and superconducting (SC) states in a Hubbard-Heisenberg model on an anisotropic triangular lattice. We use a renormalized mean field theory to study the Gutzwiller projected BCS wavefucntions. The half filled electron system is a Mott insulator at large on-site repulsion $U$, and is a superconductor at a moderate $U$. The symmetry of the SC state depends on the anisotropy, and is gapful with $d_{x^{2}-y^{2}}+i d_{x y}$ symmetry near the isotropic limit and is gapless with $d_{x^{2}-y^{2}}$ symmetry at small anisotropy ratio.

PACS numbers: $74.70 . \mathrm{Kn}, 74.20 . \mathrm{Rp}, 74.25 . \mathrm{Dw}$
\end{abstract}

Understanding of exotic properties in layered organic superconductors $\kappa$-(ET) $)_{2} X \quad(\mathrm{X}=$ anion $)$ has attracted much interest recently $\stackrel{1,2,3.4,5.6}{ }$. In these materials, $(\mathrm{ET})_{2}$ dimers are arranged in a quasi-two-dimensional (quasi2D) anisotropic triangular lattice, as schematically shown in Fig. 1(a). Since the intradimer hopping integrals are much larger than the interdimer ones, and the carrier density of the compound is one hole per dimer, the low energy electronic structure can be well approximated by a 2D Hubbard model at the half filling, where each lattice site represents a dimer ${ }^{7}$, as illustrated in Fig. 1(b) with nearest neighboring (n.n.) and next n.n. hopping integrals $t$ and $t^{\prime}$ and a strong on-site Coulomb repulsion $U$. Under the pressure, some of the compounds show a first-order transition from a Mott insulator to a superconductor. We expect the effect of $t^{\prime}$ or the geometrical frustration to be crucial to the low temperature phases. In most of $\kappa$-(ET) ${ }_{2} X$ materials, $t^{\prime} / t \sim 0.4-0.8$, the effect of geometrical frustration is less remarkable so that the ground state of the insulating phase is antiferromagnetic, similar to the high- $T_{c} \mathrm{SC}$ cuprates $8.9 \cdot 10.11 .12$. An example is the compound with $\mathrm{X}=\mathrm{Cu}\left[\mathrm{N}(\mathrm{CN})_{2}\right] \mathrm{Br}$, where $t^{\prime} / t \sim 0.6$. For systems with a larger $t^{\prime} / t$, the frustration may destroy magnetic ordering at low temperatures and the interplay between the frustration and strong correlation may lead to the possible emergence of spin liquid state or unconventional superconductivity. The compound of $X=\mathrm{Cu}_{2}(\mathrm{CN})_{3}\left(t^{\prime} / t \sim 1.06\right)$ appears to be an excellent candidate for spin liquid state without manifestation of any magnetic ordering as indicated in the NMR at low temperatures down to $32 \mathrm{mK}^{13}$, reminiscent of the resonating valence bond state proposed by Anderson ${ }^{14.15}$ in a triangular lattice system. The compound becomes $\mathrm{SC}$ under moderate hydrostatic pressure with the maximum $T_{c}$ of $3.9 \mathrm{~K}$.

The SC pairing symmetry in the layered organic conductors remains controversial. Experimentally, the NMR measurements9.10.16, the angular dependent scanning tunneling microscopy 17 and the thermal conductiv-

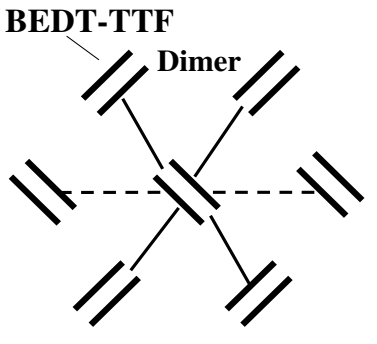

(a)

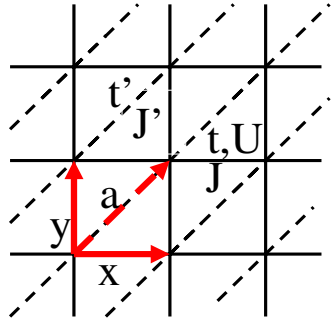

(b)
FIG. 1: (a) $\kappa$-type ET molecular arrangement within the conducting plane. (b) Anisotropic triangular lattice structure of the 2D Hubbard-Heisenberg $\left(t-t^{\prime}-J-J^{\prime}-U\right)$ model. Also shown are three unit vectors on the lattice.

ity measurements in the vortex state $\frac{18}{}$ all suggest the existence of nodes in the gap and indicate a spin singlet pairing $\mathrm{d}_{x^{2}-y^{2}}$ symmetry, similar to that of high- $\mathrm{T}_{c}$ cuprates. However, the temperature dependence of the penetration depth ${ }^{19,20}$ and of the specific heat ${ }^{21}$ seem to support a full gapped SC state. Note that there are also results consistent with a gap with nodes 22.23 . On the theoretical side, fluctuation exchange approximation for the Hubbard model shows a pure $d_{x^{2}-y^{2}}$-wave symmetry ${ }^{24}$ while the variational Monte Carlo and mean field study for a generalized $t-J$ model $\left(t-t^{\prime}-J-J^{\prime}\right.$ model $)$ suggest that $d_{x^{2}-y^{2}}+i d_{x y^{-}}(d+i d-)$ wave state is more stable $25,26,27$. Concerning the order of SC to Mott insulator transition, there are two recent studies ${ }^{28,29}$ based on variational Monte Carlo calculation by analyzing a $2 \mathrm{D}$ Hubbard model. They find a first-order SC to Mott insulator transition for intermediate $U$ and the SC state is restricted to the $d_{x^{2}-y^{2}}$-wave symmetry.

In a previous paper, we considered the competition between $d_{x^{2}-y^{2}}$-wave SC and antiferromagnetic states 
in $\kappa$-(ET) $)_{2}$ salts in the parameter region $t^{\prime} / t<0.8^{30}$. The Gutzwiller renormalized mean-field theory ${ }^{31}$ was employed to study an anisotropic Hubbard-Heisenberg model in a 2D lattice. We found a first-order transition between a Gossamer superconductor at a smaller $U$ and an antiferromagnetic insulator at a larger $U$. Our result was qualitatively consistent with major experiments. In this paper, we are primarily interested in layered organic conductor with $t^{\prime} / t \sim 1$, with the compound $X=\mathrm{Cu}_{2}(\mathrm{CN})_{3}$ in mind. Our focus will be on the Mott insulator to SC transition and the pairing symmetry of the SC state. We expect the anisotropic ratio $t^{\prime} / t$ will affect the pairing symmetry. Since the compound of $X=\mathrm{Cu}_{2}(\mathrm{CN})_{3}$ is not magnetically long range ordered, we shall neglect the antiferromagnetism in our study. We find that at half filling, the ground state is a metal for small $U$, a Mott insulator for large $U$, and a Gossamer superconductor at intermediate $U$, whose pairing is $d+i d$ near the isotropic point. A transition between different pairing symmetries may show up by tuning the model parameters.

We start with a Hubbard-Heisenberg model on an anisotropic triangular lattice,

$$
\begin{aligned}
H & =U \sum_{i} n_{i \uparrow} n_{i \downarrow}-\sum_{\langle i j\rangle \sigma} t_{i j}\left(c_{i \sigma}^{\dagger} c_{j \sigma}+\text { h.c. }\right) \\
& +\sum_{\langle i j\rangle} J_{i j} S_{i} \cdot S_{j}-\mu \sum_{i} n_{i},
\end{aligned}
$$

where $c_{i \sigma}^{\dagger}$ is to create a hole with spin $\sigma$ at site $i, S_{i}$ is a spin operator, $\mu$ is the chemical potential, and $\langle i j\rangle$ denotes a neighboring pair on the lattice. $t_{i j}=t, J_{i j}=J$ for the neighboring pairs along $x$ and $y$ directions, and $t_{i j}=t^{\prime}, J_{i j}=J^{\prime}$ for the neighboring pairs along the $a$ direction as shown in Fig. 1(b). Hereafter we set $t=1$ and use $t$ as the energy unit, and choose $J / t=1 / 3$ and consider mainly the system in the parameter range $t^{\prime} / t=0.8-1.2$. We choose $J^{\prime} / J=\left(t^{\prime} / t\right)^{2}$, consistent with the relation of $J=4 t^{2} / U$ in the large $U$ limit. We will exclusively consider the half filled case. When $t^{\prime} / t=J^{\prime} / J=1$, the model becomes isotropic. At $U \rightarrow \infty$, the model is reduced to the Heisenberg model. We remark that the Heisenberg model on an isotropic triangular lattice has a long range antiferromagnetic order with an angle of $2 \pi / 3$ between neighboring spins. However, there is the possibility to have a spin liquid state in the anisotropic Heisenberg mode ${ }^{32}$ and in the anisotropic Hubbard model ${ }^{33}$. In what follows below, we will devote to the non-magnetic states in our variational approach.

We consider a partially Gutzwiller projected BCS trial wavefunction $\stackrel{34.35,36}{ }$,

$$
\begin{aligned}
\left|\Psi_{G S}\right\rangle & =\prod_{i} P_{i}\left|\Psi_{B C S}\right\rangle \\
\left|\Psi_{B C S}\right\rangle & =\prod_{k}\left(u_{k}+v_{k} c_{k \uparrow}^{\dagger} c_{-k \downarrow}^{\dagger}\right)|0\rangle
\end{aligned}
$$

where $P_{i}=z^{n_{i}}\left(1-\alpha n_{i \uparrow} n_{i \downarrow}\right)$ is a projection operator to partially project out the doubly occupied electron states on the site $i$ and $0 \leq \alpha \leq 1$ measures the strength of the projection. $z$ is the fugacity to ensure the charge density is unchanged by projection ${ }^{34.37 .38 .39}$. Note that introduction of the fugacity does not change the previous results on the renormalized mean field theory for the Gutzwiller state for that the number of charge carriers were assumed to be the same 31 .

We then apply the Gutzwiller approximation to estimate the variational energy. In this scheme, the effect of the projection operator is taken into account by a set of renormalized factors, which are determined by statistical countings ${ }^{40.41}$. Let $\langle Q\rangle$ be the expectation value of $Q$ in the state $\left|\Psi_{G S}\right\rangle$ and $\langle Q\rangle_{0}$ be that in the state $\left|\Psi_{B C S}\right\rangle$. The Gutzwiller approximation gives,

$$
\left\langle c_{i \sigma}^{\dagger} c_{j \sigma}\right\rangle \approx g_{t}\left\langle c_{i \sigma}^{\dagger} c_{j \sigma}\right\rangle_{0}, \quad\left\langle\vec{S}_{i} \cdot \vec{S}_{j}\right\rangle \approx g_{s}\left\langle\vec{S}_{i} \cdot \vec{S}_{j}\right\rangle_{0}
$$

where $g_{t}$ and $g_{s}$ are the Gutzwiller renormalized coefficients and can be derived as follows $\frac{35}{3}$,

$$
\begin{aligned}
& g_{t}=\frac{(n-2 d)(\sqrt{d}+\sqrt{1-n+d})^{2}}{(1-n / 2) n} \\
& g_{s}=\left[\frac{(n-2 d)}{(1-n / 2) n}\right]^{2}
\end{aligned}
$$

where $n$ is the average electron number and $d=\left\langle n_{i \uparrow} n_{i \downarrow}\right\rangle$ is the average electron double occupation number. There is one-to-one correspondence between $\alpha$ and $d$. Within the Gutzwiller approximation, we have,

$$
(1-\alpha)^{2}=\frac{d(1-n+d)}{(n / 2-d)^{2}}
$$

In terms of the renormalized coefficients, we can get the effective Hamiltonian,

$$
\begin{aligned}
H_{e f f} & =U d-g_{t} \sum_{\langle i j\rangle \sigma} t_{i j} c_{i \sigma}^{\dagger} c_{j \sigma}+h . c . \\
& +g_{s} \sum_{\langle i j\rangle} J_{i j} S_{i} \cdot S_{j}-\mu \sum_{i} n_{i} .
\end{aligned}
$$

The variation of the projected state $\left|\Psi_{G S}\right\rangle$ for $H$ in (1) is thus reduced to the variation of the unprojected state $\left|\Psi_{B C S}\right\rangle$ for $H_{\text {eff }}$. Note that the Gutzwiller projection on a BCS state may change the average number of electrons of the state.

To proceed further, we introduce two types of mean fields, $(\tau= \pm \hat{x}, \pm \hat{y}, \pm \hat{a})$

$$
\begin{aligned}
\Delta_{i, i+\tau} & =\left\langle c_{i \downarrow} c_{i+\tau \uparrow}-c_{i \uparrow} c_{i+\tau \downarrow}\right\rangle_{0} \\
\chi_{i, i+\tau} & =\sum_{\sigma}\left\langle c_{i \sigma}^{\dagger} c_{i+\tau \sigma}\right\rangle_{0} .
\end{aligned}
$$

We consider the translational invariant state with even parity, $\Delta_{i, i+\tau}=\Delta_{i+\tau, i}=\Delta_{\tau}$, and $\chi_{i, i+\tau}=\chi_{\tau}$. Applying the mean field theory to $H_{e f f}$, we obtain,

$$
\begin{aligned}
\left|u_{\vec{k}}\right|^{2} & =\left(E_{\vec{k}}+\varepsilon_{\vec{k}}\right) / 2 E_{\vec{k}}, \\
\left|v_{\vec{k}}\right|^{2} & =\left(E_{\vec{k}}-\varepsilon_{\vec{k}}\right) / 2 E_{\vec{k}}, \\
E_{\vec{k}} & =\sqrt{\varepsilon_{\vec{k}}^{2}+\left|F_{\vec{k}}\right|^{2}},
\end{aligned}
$$


where

$$
\begin{aligned}
\varepsilon_{\vec{k}} & =-g_{t} \sum_{\tau} 2 t_{\tau} \cos k_{\tau}-\mu-\frac{3 g_{s}}{4} \sum_{\tau} J_{\tau} \chi_{\tau} \cos k_{\tau} \\
F_{\vec{k}} & =\frac{3}{4} g_{s} \sum_{\tau} \Delta_{\tau} \cos k_{\tau}
\end{aligned}
$$

where $k_{\tau}=\vec{k} \cdot \vec{\tau}$, and the sum in $\tau$ runs over the three unit vectors. In particular, we assume $\Delta_{a}$ to be real, and $\Delta_{x}$ and $\Delta_{y}$ to have a phase $\theta$ and $-\theta$ relative to $\Delta_{a}$, respectively

$$
\Delta_{a}=\left|\Delta_{a}\right|, \Delta_{x}=\left|\Delta_{x}\right| e^{-i \theta}, \Delta_{y}=\left|\Delta_{y}\right| e^{i \theta} .
$$

The case of $\Delta_{a}=0$ and $\theta=\pi / 2$ corresponds to the pure $d_{x^{2}-y^{2}}$ state. In the case $\theta=0$, we have an extended $s$ wave state. In general, $\Delta_{a} \neq 0$ and $\theta \neq \pi / 2$, we have $d+i d$ symmetry ${ }^{26}$.

The self-consistent equations are given as

$$
\begin{aligned}
\Delta_{\tau} & =\frac{1}{N} \sum_{\vec{k}} \cos k_{\tau} F_{\vec{k}} / E_{\vec{k}} \\
\chi_{\tau} & =-\frac{1}{N} \sum_{\vec{k}} \cos k_{\tau} \varepsilon_{\vec{k}} / E_{\vec{k}} \\
\delta & =\frac{1}{N} \sum_{\vec{k}} \varepsilon_{\vec{k}} / E_{\vec{k}}
\end{aligned}
$$

where the last equation is on the number of holes which is zero at the half filled of our interest here, and $N$ is the number of total lattice sites and the sum in $\vec{k}$ runs over the first Brillouin zone. The ground state energy is

$$
E=U d-2 g_{t} \sum_{\tau} t_{\tau} \chi_{\tau}-\frac{3 g_{s}}{8} \sum_{\tau} J_{\tau}\left(\left|\Delta_{\tau}\right|^{2}+\left|\chi_{\tau}\right|^{2}\right)
$$

All the mean fields are determined self-consistently for each set of model parameters $U, t^{\prime} / t$ and a given variational parameter $d$, from which we obtain the lowest energy state with respect to the variation of $d$.

We first examine the evolution of the ground state by varying the anisotropy ratio $t^{\prime} / t$. The anisotropy ratio is a measure of the geometrical frustration, which modifies the shape of Fermi surface and therefore plays an important role in determining the competition between Mott insulator and various SC states. As shown in our previous study ${ }^{30}$, for small anisotropy $t^{\prime} / t$, the ground state is an antiferromagnetic insulator at large $U$ and a Gossamer superconductor at medium $U$, followed by a normal metallic state at small $U$, and the transition between Mott insulator and SC state belongs to the first order. We expect the physics of the model in a triangular lattice with large anisotropy may be qualitatively different from that of a less anisotropic lattice. For large $t^{\prime} / t$, the antiferromagnetic state may be degraded into spin liquid state and time-reversal symmetry broken SC state may show up as well. We note that the transition from the Mott insulator to SC state remains first order.

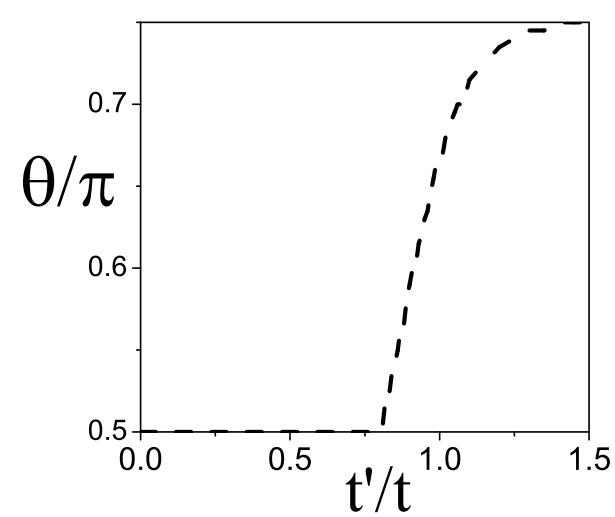

FIG. 2: The relative phase of pairing parameter as a function of $t^{\prime} / t$.

In our calculation, the relative phase $\theta$ of pairing parameter is obtained by the minimization of the total energy. Due to the numerical difficulty to determine the exact value of $\theta$ for small but finite $d$, we present the results in Fig. 2 for the case $d=0$. The approximate critical value of $\left(t^{\prime} / t\right)_{c} \sim 0.81$ distinguishes the different pairing symmetry region ${ }^{42}$, the $\mathrm{SC}$ order is always with $d_{x^{2}-y^{2}}$ symmetry $(\theta=\pi / 2)$ for $t^{\prime} / t<\left(t^{\prime} / t\right)_{c}$ while imaginary part of pairing parameter emerges and the relative phase $\theta$ exceeds $\pi / 2$ for $t^{\prime} / t>\left(t^{\prime} / t\right)_{c}$. The SC order parameter with nonzero imaginary part corresponds to $d+i d$-wave state which breaks time-reversal symmetry. The presence of such state may result in the appearance of excitation gaps all over the Fermi surface. This timereversal symmetry broken state might be detectable by using muon-spin rotation measurements ${ }^{43}$. It is interesting to note that when $t^{\prime}=t$ and $J^{\prime}=J$, the relative phase $\theta$ is equal to $2 \pi / 3$ where all the three amplitudes become symmetrical and we have $\Delta=\Delta_{x}=\Delta_{y}=\Delta_{a}$ and $\chi=\chi_{x}=\chi_{y}=\chi_{a}$. This special pairing symmetry is due to the geometrical symmetry of the isotropic triangular lattice. This result is fully consistent with other works for a $t$ - $J$ model on a triangular lattice ${ }^{26.27}$. The continuous change of the relative phase $\theta$ is found by further increasing the anisotropic parameter $t^{\prime} / t$. For $t^{\prime} / t>1.3$, we find that the value of $\theta$ saturates to $3 \pi / 4$.

Our detailed results for $t^{\prime} / t=1$ are presented in Fig. 3. Here we denote the SC order parameter as $\Delta_{S C}=\left\langle c_{i \downarrow} c_{j \uparrow}-c_{i \uparrow} c_{j \downarrow}\right\rangle$. Within the Gutzwiller approximation, $\Delta_{S C}$ can be expressed as $\Delta_{S C}=g_{t} \Delta$. The effect of large $U$ may suppress the double occupancy $d$ and stabilize the Mott insulating state. As shown in Fig. 3(a), it is obvious that there exists a critical $U_{c}(\sim 12.2 t)$ to separate a $\mathrm{SC}$ state at a small $U$ from a Mott insulator at a large $U$, and the transition is first order with no coexistence of the two phases. At $U<U_{c}$, both $\Delta$ and $\Delta_{S C}$ increase monotonically as $U$ increases. $\Delta_{S C}$ is smaller than $\Delta$ because of the prefactor $g_{t}<1$. At the critical point, $\Delta$ increases to its maximum while $\Delta_{S C}$ jumps to 

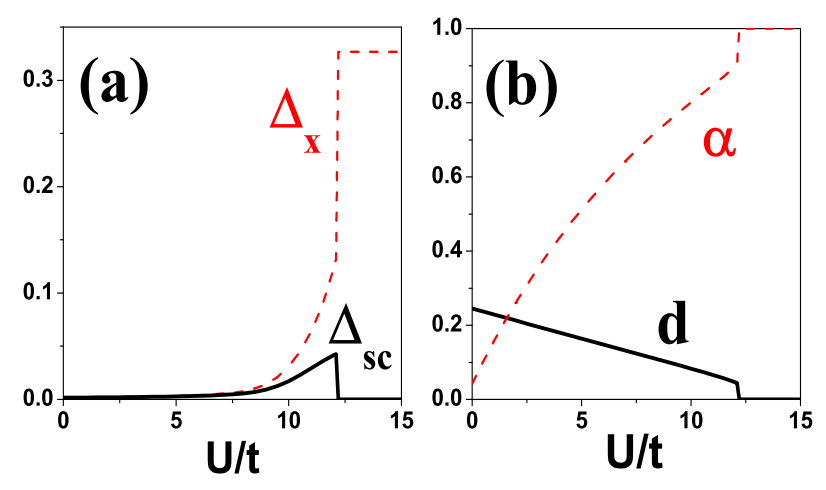

FIG. 3: Pairing amplitude $\Delta_{x}$ and its SC order parameter $\Delta_{x}^{S C}(\mathrm{a})$, and electron double occupancy number $d$ and the projection parameter $\alpha(\mathrm{b})$, as functions of $U / t$ for $t^{\prime} / t=1$.

zero suddenly. At $U>U_{c}$, the system becomes an insulator with $d=0$, or indicates the full projection in the insulating phase. The projection parameter $\alpha$ reaches its maximum in the SC phase followed by a discontinuous jump to 1 at $U=U_{c}$. All these features suggest that the first order transition is robust.

Another interesting feature reveals that in the SC phase just below $U_{c}$, the double occupation number $d$ is quite small $(\sim 0.04)$, while $\alpha$ is large $(\sim 0.91)$. In accordance with Laughlin's proposal of Gossamer superconductivity ${ }^{34}$, very tiny superfluidity density appears in the SC state. It is obvious that the SC state in this system can be well characterized by the Gossamer superconductor. All the previous studies for less frustrated system show the presence of appreciable (not so tiny) superfluid density. In our present study, it is clear that the effect of strong geometrically frustration may remarkably suppress the superfluid density of the SC state $\underline{44}$.
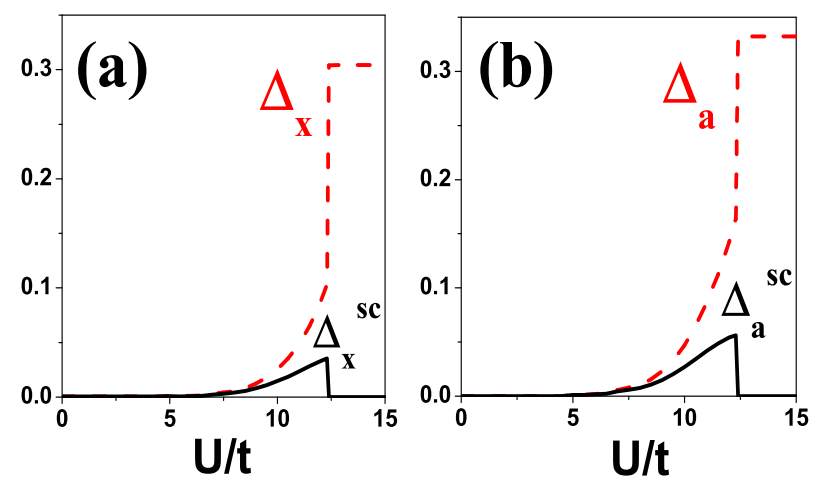

FIG. 4: Pairing amplitudes and its SC order parameter as functions of $U / t$ for $t^{\prime} / t=1.06$. (a) $\Delta_{x}$ and $\Delta_{x}^{S C}$. (b) $\Delta_{a}$ and $\Delta_{a}^{S C}$.

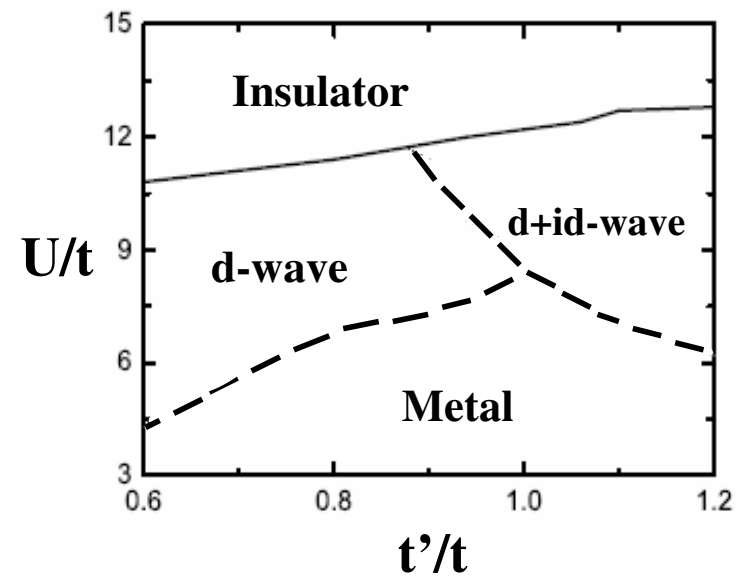

FIG. 5: Phase diagram in parameter space $t^{\prime} / t$ and $U / t$.

Next we study the particular case for the compound $\kappa$ $(\mathrm{ET})_{2} \mathrm{Cu}_{2}(\mathrm{CN})_{3}$ which has a large degree of geometrical frustration $t^{\prime} / t \sim 1.06$. One expects the general features are similar to the $t^{\prime} / t=1$ case. As depicted in Fig. 4 , we notice two features which are in contrast to the $t^{\prime} / t=1$ case. For the anisotropic parameter $t^{\prime} / t>1$, the amplitude of $\Delta_{a}$ becomes larger than that of $\Delta_{x}$ while the SC order parameter of $\Delta_{a}^{S C}$ is more pronounced than that of $\Delta_{x}^{S C}$. In other words, the relative phase of SC order parameter $\theta$ may deviate from the symmetrical value $2 \pi / 3$ and will be around $0.70 \pi$. Another distinctive feature shows that the Mott transition point $U_{c}$ strongly depends on lattice structure anisotropy $t^{\prime} / t$. Due to the competing nature between Mott insulating phase and the SC phase, the increasing of geometrical frustration $t^{\prime} / t$ may eventually destabilize the Mott insulating phase which may lead to the Mott transition and result in a SC phase. We find that the critical value increases slightly from $U_{c}=12.2$ for $t^{\prime} / t=1$ to $U_{c}=12.5$ for $t^{\prime} / t=1.06$. This result suggests that the increasing of $t^{\prime} / t$ may effectively enhance the critical value $U_{c}$.

Fig. 5 displays the phase diagram in the parameter space of $t^{\prime} / t$ and $U / t$. It is technically difficult to determine precisely the critical boundaries between various phases due to the presence of degenerate states at half filling for highly frustrated system. In practice, we define a paramagnetic metallic phase if both $\Delta_{x}$ and $\Delta_{a}$ are less than 0.01 and a $d+i d$ state if $\Delta_{a}>0.01$. At very small $\Delta$, the energy discrepancy between these phases becomes indistinguishable in our calculation. We use dashed lines to represent the phase boundaries with very small energy differences. The solid line denotes the first-order phase boundary between SC phase and Mott insulating state. According to our phase diagram, there exists four distinct phases. The system is in the Mott insulating phase at large $U$ and small $t^{\prime}$, the paramagnetic metallic phase at small $U$ and large $t^{\prime}$, and the two possible SC phases at the intermediate parameter region. For small $t^{\prime} / t$, the phase diagram is consistent with our previous 
study ${ }^{30}$. Note here that the time-reversal symmetry broken $d+i d$ state is preferable for large $t^{\prime} / t$ while the pure $d_{x^{2}-y^{2}}$-wave state is more stable for small $t^{\prime} / t$. Since the effect of pressure may increase $U / t$ and/or to increase $t^{\prime} / t$, it is quite plausible that a flow of parameters $U / t$ and $t^{\prime} / t$ may lead to a pairing symmetry transition from $d_{x^{2}+y^{2}}$ to $d+i d$ state. In a very recent paper ${ }^{45}$, the transition from $d+i d$ to $d$-wave is also indicated. Two recent investigations ${ }^{28.29}$ addressed the first-order Mott transition by applying variational Monte Carlo method for the layered organic superconductors by analyzing a two-dimensional Hubbard model. By using a modified variational wavefunction with doublon-holon binding factors, they found an unconventional SC ground state for medium $U$, sandwiched between a normal metal at weak coupling and a spin liquid at larger coupling. A firstorder Mott transition takes place at certain value of $U$. Our renormalized mean field theory can take into account approximately the effect of Gutzwiller projection and the results agree qualitatively with theirs. It is well known that the Brinkman-Rice ${ }^{46}$ type metal-insulator transition for Hubbard model belongs to the second-order. It seems to us that the extra spin exchange term in the HubbardHeisenberg model makes much difference and changes the
Gossamer SC to Mott insulator transition to be firstorder.

In summary, we have utilized the Gutzwiller mean-field method to study the $t-t^{\prime}-J-J^{\prime}-U$ model on a highly frustrated anisotropic triangular lattice. We find that the pure $d_{x^{2}-y^{2}}$ state with nodal structure is stable if the geometrical frustration parameter $t^{\prime} / t<\left(t^{\prime} / t\right)_{c}$ while the gapped $d+i d$ state is more preferable for $t^{\prime} / t>\left(t^{\prime} / t\right)_{c}$ in the SC phase. In the case of $t^{\prime} / t=1$, the relative phase of the SC order parameter is equal to $2 \pi / 3$ which reflects the geometrical symmetry of isotropic triangular lattice. For a fixed $t^{\prime} / t$, the ground state is found to be a paramagnetic metal at small on-site Coulomb repulsion $U$; a Mott insulator at large $U$; and a superconductor at intermediate $U$. This SC state can be well described by the Gossamer superconductivity with tiny superfluid density. The transition between SC and insulating phases belongs to first order. Our results may shed light on the understanding of the exotic features of $\kappa-(\mathrm{ET})_{2} \mathrm{Cu}_{2}(\mathrm{CN})_{3}$, which undergoes a possible spin liquid to superconductor transition at half filling under pressure.

This work is supported by the RGC grants of Hong Kong SAR government, and seed funding grant from the University of Hong Kong.
1 D. Jerome, Science 252, 1509 (1991).

2 R. H. Mckenzie, Science 278, 820 (1997); Comments Condens. Matter Phys. 18,309 (1998).

3 T. Ishiguro, K. Yamaji, and G. Saito, Organic Superconductors (Springer, Berlin, 1998), 2nd ed.

${ }^{4}$ M. Lang and J. Mueller, cond-mat/0302157

${ }^{5}$ K. Kuroki, cond-mat/0601060

${ }^{6}$ F. Kagawa, K. Miyagawa, and K. Kanoda, Nature 436, 534 (2005).

7 H. Kino and H. Fukuyama, J. Phys. Soc. Jpn. 64, 2726 (1995).

8 K. Miyagawa, A. Kawamoto, Y. Nakazawa, and K. Kanoda, Phys. Rev. Lett. 75, 1174 (1995).

${ }^{9}$ H. Mayaffre, P. Wzietek, D. Jerome, C. Lenoir, and P. Batail, Phys. Rev. Lett. 754122 (1995).

10 S. M. De Soto, C. P. Slichter, A. M. Kini, H. H. Wang, U. Geiser, and J. M. Williams, Phys. Rev. B 52, 10364 (1995).

11 S. Lefebvre, P. Wzietek, S. Brown, C. Bourbonnais, D. Jerome, C. Meziere, M. Fourmigue, and P. Batail, Phys. Rev. Lett. 85, 5420 (2000).

12 K. Miyagawa, A. Kawamoto, and K. Kanoda, Phys. Rev. Lett. 89017003 (2002).

13 Y. Shimizu, K. Miyagawa, K. Kanoda, M. Maesato and G. Saito, Phys. Rev. Lett. 91107001 (2003).

14 P. W. Anderson, Mater. Res. Bull. 8, 153 (1973).

15 P.W. Anderson, P.A Lee, M. Randeria, T.M. Rice, N. Trivedi and F.C. Zhang, J. Phys. Cond. Matt. 24, R755 (2004).

16 K. Kanoda, K. Miyagawa, A. Kawamoto, and Y. Nakazawa, Phys. Rev. B 54, 76 (1996).

17 T. Arai, K. Ichimura, K. Nomura, S. Takasaki, J. Yamada, S. Nakatsuji, and H. Anzai, Phys. Rev. B 63, 104518
(2001).

18 K. Izawa, H. Yamaguchi, T. Sasaki, and Y. Matsuda, Phys. Rev. Lett. 88, 027002 (2001).

19 M. Lang, N. Toyota, T. Sasaki, and H. Sato, Phys. Rev. Lett. 69, 1443 (1992).

20 M. Dressel, O. Klein, G.Grüner, K.D. Carlson, H.H. Wang, and J.M. Williams, Phys. Rev. B 50, 13603 (1994).

21 H. Elsinger, J. Wosnitza, S. Wanka, J. Hagel, D. Schweitzer, and W. Strunz, Phys. Rev. Lett. 84, 6098 (2000).

22 K. Kanoda, K. Akiba, K. Suzuki, T. Takahashi, and G. Saito, Phys. Rev. Lett. 65, 1271 (1990).

23 A. Carrington, I.J. Bonalde, R. Prozorov, R.W. Giannetta, A.M. Kini, J. Schlueter, H.H. Wang, U. Geiser, and J.M. Williams, Phys. Rev. Lett. 834172 (1999).

24 J. Schmalian, Phys. Rev. Lett. 814232 (1998); H. Kino and H. Kontani, J. Phys. Soc. Jpn. 673691 (1998); H. Kondo and T. Moriya, J. Phys. Soc. Jpn. 673695 (1998).

${ }^{25}$ T. Watanabe, H. Yokoyama, Y. Tanaka, J. Inoue and M. Ogata, J. Phys. Soc. Jpn. 73, 3404 (2004).

${ }^{26}$ M. Ogata, J. Phys. Soc. Jpn. 72, 1839 (2003).

27 B. Kumar and B.S. Shastry, Phys. Rev. B 68, 104508 (2003); G. Baskaran, Phys. Rev. Lett. 91, 097003 (2003).

28 J. Liu, J. Schmalian and N. Trivedi, Phys. Rev. Lett. 94, 127003 (2005)

29 T. Watanabe, H. Yokoyama, Y. Tanaka and J. Inoue, cond-mat/0602098

30 J.Y. Gan, Y. Chen, Z.B. Su and F.C. Zhang, Phys. Rev. Lett. 94, 067005 (2005).

31 F.C. Zhang, C. Gros, T.M. Rice, and H. Shiba, Supercond. Sci. Technol. 1, 36 (1988).

32 A. E. Trumper, Phys. Rev. B 60, 2987 (1999); Zheng Weihong, R. H. McKenzie, and R. P. Singh, ibid. 59, 14367 
(1999).

33 H. Morita, S. Watanabe, and M. Imada, J. Phys. Soc. Jpn. 71, 2109 (2002).

${ }^{34}$ R. Laughlin, cond-mat/0209269

35 F.C. Zhang, Phys. Rev. Lett. 90, 207002 (2003); J.Y. Gan, F.C. Zhang, and Z.B. Su, Phys. Rev. B 71, 014508 (2005).

36 B.J. Powell and R.H. McKenzie, Phys. Rev. Lett. 94, 047004 (2005).

37 P. W. Anderson and N. P. Ong, cond-mat/0405518 (unpublished).

38 B. Edegger, N. Fukushima, C. Gros, and V. N. Muthukumar, Phys. Rev. B 72, 134504 (2005).

39 Q. H. Wang, Z. D. Wang, Y. Chen, and F. C. Zhang, Phys. Rev. B 73, 092507 (2006).
40 M. C. Gutzwiller, Phys. Rev. 137, A1726 (1965).

41 D. Vollhardt, Rev. Mod. Phys. 56, 99 (1984).

${ }^{42}$ In reference [26], one obtains the critical value $\left(t^{\prime} / t\right)_{c} \sim$ 0.675 . The discrepancy is due to the fact that in their calculation, the double occupation is fixed to zero and different parameters as $J / t=0.3$ and $J^{\prime} / J=t^{\prime} / t$ are used.

43 F.L. Pratt, S.J. Blundell, T. Lancaster, M.L. Brooks, S.L. Lee, N. Toyota and T. Sasaki, Synth. Met. 152, 417 (2006).

44 B.J. Powell and R.H. McKenzie, J. Phys.: Condens. Matter. 16, L367 (2004); F. L. Pratt and S.J. Blundell, Phys. Rev. Lett. 94, 097006 (2005).

45 B.J. Powell, cond-mat/0603057

46 W. Brinkman and T.M. Rice, Phys. Rev. B 2, 4302 (1970). 